\title{
Impact of sensor metal thickness on microwave spectroscopy sensitivity for individual particles and biological cells analysis
}

\author{
W. Chen, D. Dubuc, K. Grenier \\ LAAS-CNRS, 7. Av. du Colonel Roche, F-31031 Toulouse
}

\begin{abstract}
This paper focuses on evaluating the impact of metal thickness of a microwave coplanar based sensor dedicated to the microwave dielectric spectroscopy of single particles and individual biological cells. A sensitivity study has therefore been achieved for metal thicknesses comprised between 0.3 and $20 \mu \mathrm{m}$. After the validation of electromagnetic simulations with measurements of $10 \mu \mathrm{m}-$ diameter polystyrene bead, both capacitive and conductive contrasts have been defined for the different metal thickness of the sensor. The maximal sensitivity improvement is therefore achieved for a thickness value similar to the diameter of the particle or cell to measure. Capacitive and conductive contrasts are increased by a factor 2.4 and 1.75 respectively. The study leads consequently to an important design and fabrication rule of such a sensor.
\end{abstract}

Index Terms - Microwave, coplanar waveguide, biosensor, dielectric spectroscopy, sensitivity, biological cell.

\section{INTRODUCTION}

The measurement of biological cells constitutes a routine step in many biological investigations. Actual techniques used by biologists are mainly based on optical staining or fluorescent markers, which provide very precise and efficient molecular and cellular observations. Within this context, microwave dielectric spectroscopy for cellular analysis constitutes a new and attractive method, due to the lack of cell preparation and manipulation, without any addition of chemicals, which may interfere with other cells constituents [1].

Therefore, investigations have been done in flow [2] or in static, with broadband [3] or narrowband sensors architectures [4]-[6]. To enhance sensitivity, design optimizations are now required [7]. This paper focuses on the impact of the metal thickness of the coplanar waveguide (CPW) on the sensitivity of a broadband single cell sensor.

After a rapid description of the microwave biosensor given in section II, section III presents the validation of the electromagnetic simulations of the RF biosensor compare to measurements of polystyrene beads. A sensitivity study in regards to the coplanar waveguide metal thickness is then given in the next section. Finally, the final section is dedicated to the discussion of the results based on electromagnetic field distributions.

\section{BRIEF DESCRIPTION OF THE MICROWAVE BIOSENSOR}

Fig. 1 presents the schematic and photography of the microwave biosensor used to characterize different particles or biological cells in a liquid host medium.

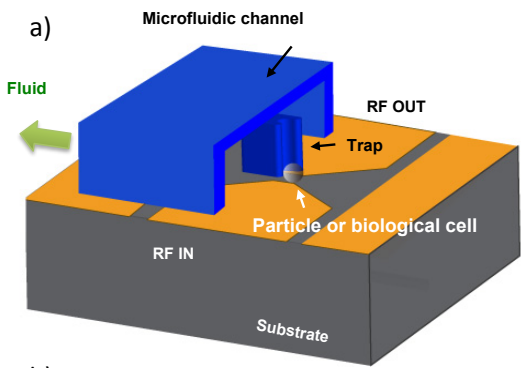

b)

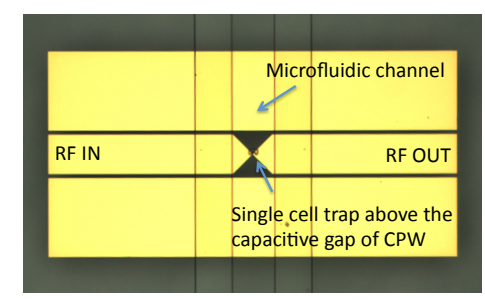

Fig. 1. a) Schematic and b) photography of the microwave biosensor dedicated to the microwave spectroscopy of individual particle or biological cell.

The sensor includes a coplanar waveguide with a capacitive gap in the center, employed to focalize the electromagnetic field within the particles or cells placed above. Those ones are trapped with a mechanical blocker localized inside a microfluidic channel. This sensor has previously been validated with polystyrene beads and biological cells [3], [7], with more related details.

\section{VALIDATION OF THE ElECTROMAGNETIC SiMULATIONS WITH POLYSTYRENE BEADS MEASUREMENTS}

Electromagnetic simulations of this sensor have been performed with the tri-dimensional software $\mathrm{HFSS}^{\odot}$, when 
loaded with polystyrene beads in de-ionized water. These simulations have been validated with the measurements of polystyrene beads, which present a diameter of $10 \mu \mathrm{m}$, for two widths of the capacitive gap, 5 and $10 \mu \mathrm{m}$ respectively.

A method [3] has been established to extract the capacitive and conductive contrasts of particle or biological cell of a host liquid medium in such a structure, with equations reminded below.

$$
\begin{aligned}
& \Delta \mathrm{C}=\mathrm{C}_{\text {particle }}-\mathrm{C}_{\text {DI-water }} \\
& \Delta \mathrm{G}=\mathrm{G}_{\text {particle }}-\mathrm{G}_{\text {DI-water }}
\end{aligned}
$$

The simulated and measured results on the capacitive contrast are presented in Fig. 2.

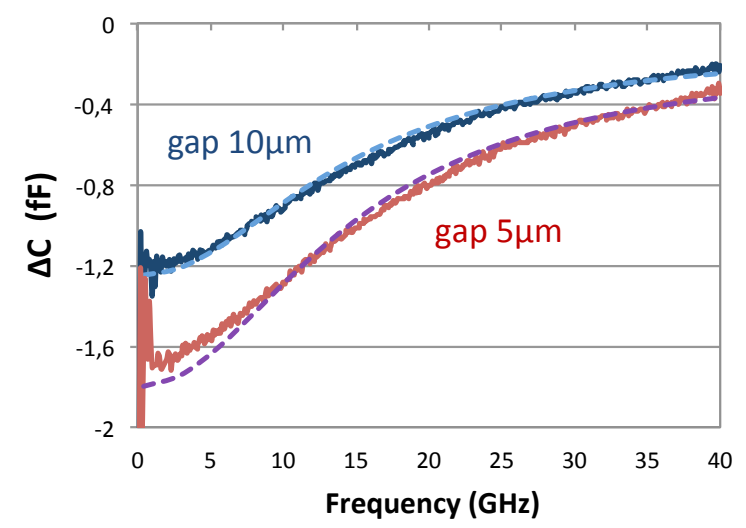

Fig. 2. Simulated and measured capacitive contrasts of polystyrene beads of $10 \mu \mathrm{m}$ diameter for a capacitive sensor gap of $10 \mu \mathrm{m}$ width.

Dashed lines correspond to the electromagnetic simulations, whereas the plain lines are related for beads measurements. Blue and red/pink curves are attributed to a capacitive gap sensor of 10 and $5 \mu \mathrm{m}$ widths respectively. An excellent agreement between simulations and measurements for the two configurations has been obtained and validates therefore the applied methodology.

With this validation, further sensitivity investigations of the sensor have consequently been possible. We focus in this paper on the impact of the CPW gold thickness on the performances.

\section{IV . ELECTROMAGNETIC SIMULATIONS OF THE RF BIOSENSOR WITH DIFFERENT GOLD WAVEGUIDE THICKNESSES}

The metallization of the actual sensor is thin and composed of a flash of titanium with a gold layer of 0.3 $\mu \mathrm{m}$ thick. Measurements of section III have been performed with sensors, which exhibit such a metal thickness.

Electromagnetic simulations have then been conducted to evaluate the impact of the sensor metal thickness on the capacitive and conductive contrasts. Polystyrene beads with a diameter of $10 \mu \mathrm{m}$ have been considered for the study, as they present a size close to biological cells previously measured with this type of sensor [3], [7]. The microwave sensor has been simulated with metal thicknesses of $3,5,10,15$ and $20 \mu \mathrm{m}$. Corresponding capacitive and conductive contrasts are given in Fig. 3 and Fig. 4 respectively.

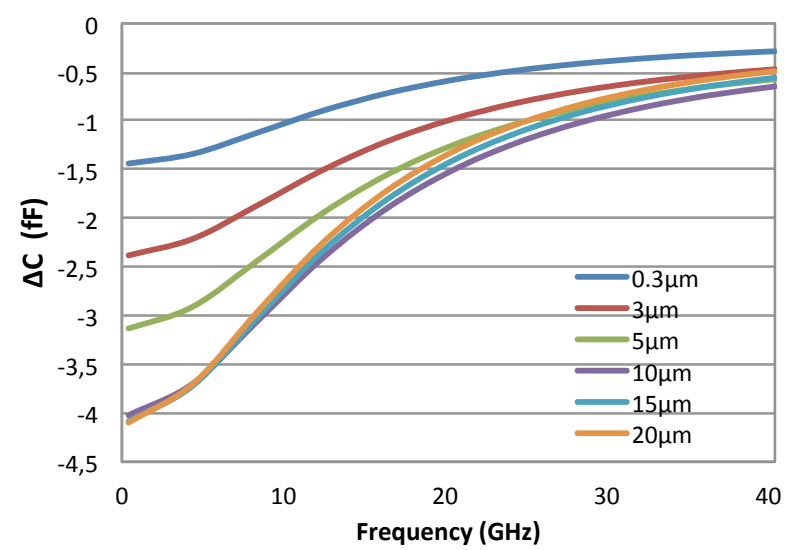

Fig. 3. Capacitive contrast of a polystyrene bead of $10 \mathrm{um}$ of diameter for gold waveguide thicknesses comprised between 0.3 um and 20 um.

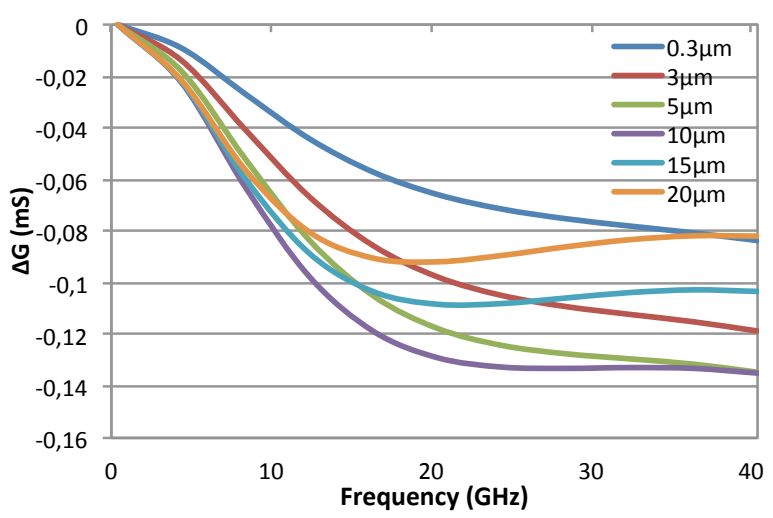

Fig. 4. Conductive contrast of a polystyrene bead of 10 um of diameter for gold waveguide thicknesses comprised between 0.3 um and $20 \mathrm{um}$.

Results from Fig. 3 presents a progressive increase of the capacitive contrast, as thickness rises. The capacitive contrast may consequently be improved by a factor of 2.4 compare to the initial one with the lowest metal thickness of $0.3 \mu \mathrm{m}$. This constitutes a major sensitivity enhancement. 


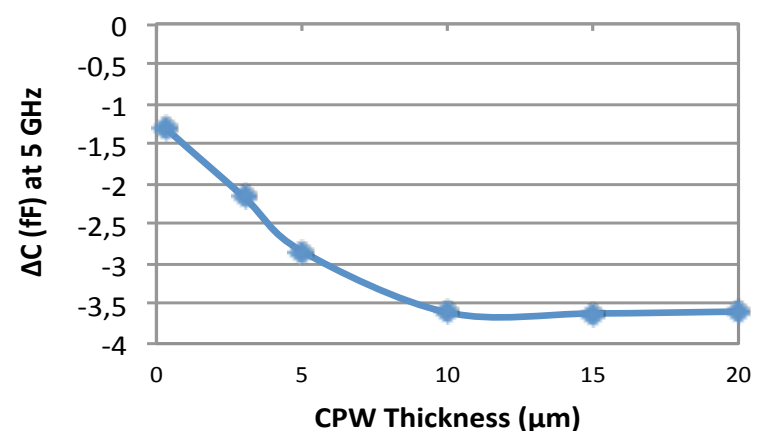

Fig. 5. Variation of the capacitive contrast versus gold CPW thickness.

Moreover, if only one frequency is considered, $5 \mathrm{GHz}$ as indicated in Fig. 5, the capacitive contrast saturates for a metal thickness of $10 \mu \mathrm{m}$. A further increase of thickness is consequently useless for this dielectric readout.

As far as the conductive contrast is concerned, the behavior is not as clear as for the capacitive contrast, which exhibited a progressive rise of the contrast with a final saturation after a threshold value. For a better visualization, Fig. 6 presents this contrast at $40 \mathrm{GHz}$ versus the metal thickness.

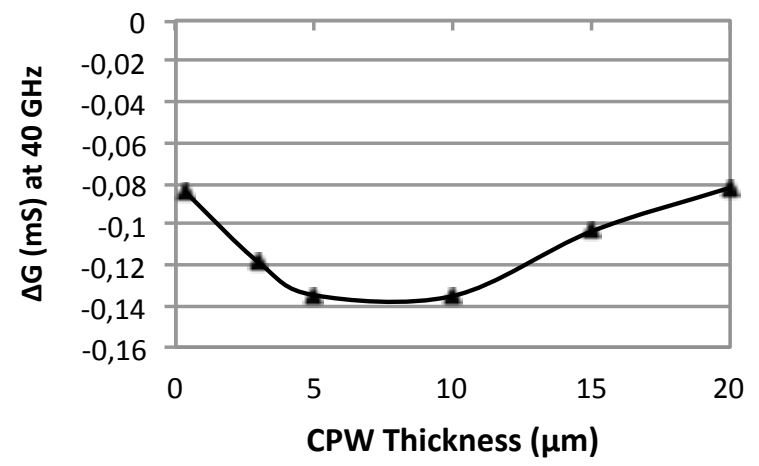

Fig. 6. Variation of the conductive contrast versus gold CPW thickness.

An improvement of the conductive contrast is first obtained for a metallization of $5 \mu \mathrm{m}$ thick and saturates up to $10 \mu \mathrm{m}$. The contrast is enhanced by a factor 1.75 . After this threshold value, the contrast progressively decreases versus the CPW thickness to reach the initial value of $0.08 \mathrm{mS}$.

Both capacitive and conductive behaviors lead consequently to the same observation: the contrasts may be improved and optimized for a gold thickness similar to the size of the polystyrene bead (10 $\mu \mathrm{m}$ in this case).
For a better understanding of these results, the electromagnetic field distributions of all cases have been extracted and are presented in the next section.

\section{ELECTROMAGNETIC FIELD DistRIBUTION DEPENDING ON GOLD WAVEGUIDE THICKNESS}

Fig. 7 presents the electromagnetic (EM) field distribution within the sensor. The localization of the bead is highlighted with a dashed circle of $10 \mu \mathrm{m}$ of diameter. The walls of the gold metallization are also visible.

One may observe a progressive increase of the maximum EM field proportion in red localized in the bead for metal thicknesses ranging from 0.3 to $10 \mu \mathrm{m}$.
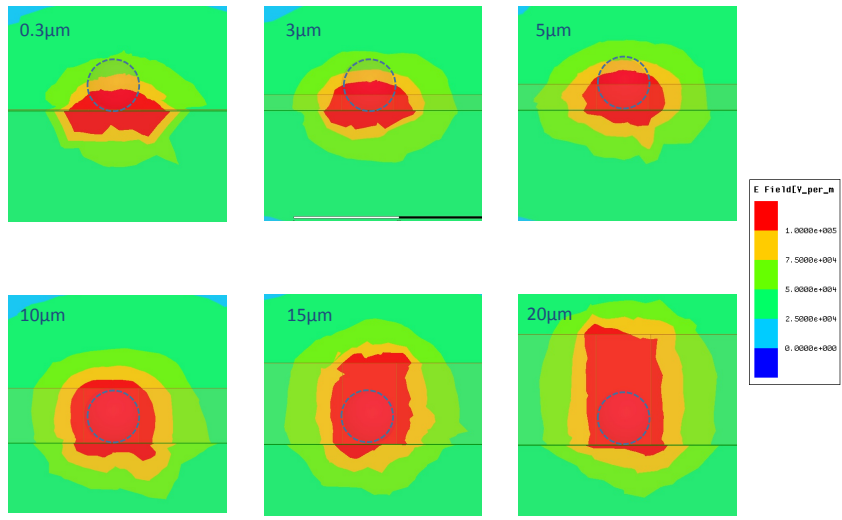

Fig. 7 EM field distribution in the sensor and bead for different CPW metal thicknesses (for a bead's diameter: $10 \mu \mathrm{m}$ and a capacitive gap of $10 \mu \mathrm{m})$.

The $10 \mu \mathrm{m}$ gold thickness permits therefore to completely localize the EM field in the bead of a similar size.

This is also confirmed by the quantitative results of Table I, which presents the proportion of EM field in the bead compare to EM field in the channel.

Table I Field concentration in the bead for metal CPW thickness comprised between 0.3 and $20 \mu \mathrm{m}$.

\begin{tabular}{|c|c|}
\hline CPW thickness & EM field in bead / EM field in the channel \\
\hline $0.3 \mu \mathrm{m}$ & $0.32 \%$ \\
\hline $3 \mu \mathrm{m}$ & $0.41 \%$ \\
\hline $5 \mu \mathrm{m}$ & $0.52 \%$ \\
\hline $10 \mu \mathrm{m}$ & $0.65 \%$ \\
\hline $15 \mu \mathrm{m}$ & $0.54 \%$ \\
\hline $20 \mu \mathrm{m}$ & $0.46 \%$ \\
\hline
\end{tabular}

The maximal concentration of EM field in the bead compared to the part in the channel is reached for the 10 $\mu \mathrm{m}$ thick gold layer with a $0.65 \%$ proportion. 


\section{CONCLUSIONS}

The impact of the metal coplanar waveguide thickness has been evaluated for a sensor dedicated to the broadband microwave spectroscopy of single particles or biological cells to enhance sensitivity. Therefore, a thickness of waveguide similar to the size of the particle diameter permits to maximize both capacitive and conductive contrasts. Improvements by a factor of 2.4 and 1.75 for the capacitive and conductive contrasts respectively have been reached. Moreover, a higher metal thickness does not degrade the capacitive contrasts, while the conductive one is finally lowered.

This study therefore contributes to define a new design and fabrication rule of such a type of sensor, related to metal thickness for enhanced sensitivity.

\section{ACKNOWLEDGEMENT}

The authors wish to acknowledge the RENATECH network for supporting the microfabrication of the device in the LAAS-CNRS clean room.

\section{REFERENCES}

[1] K. Grenier, D. Dubuc, T. Chen, F. Artis, T. Chrétiennot, M. Poupot, J-J. Fournié, 'Recent Advances in Microwavebased Dielectric Spectroscopy at the Cellular Level for Cancer Investigations', IEEE T-MTT, Vol. 61, Issue 5, Part 2, 2013, pp. 2023-2030

[2] G.A. Ferrier, S.F. Romanuik, D.J. Thomson, G.E. Bridges, M.R. Freeman, "A microwave interferometric system for simultaneous actuation and detection of single biological cells," Lab on a Chip, vol. 9, pp. 3406-3412, 2009.

[3] T. Chen et al, Microwave biosensor dedicated to the dielectric spectroscopy of a single alive biological cell in its culture medium, IEEE IMS (2013) 978-1-4673-2141.

[4] Y. Yang, P. Wang et al., "Distinguishing the viability of a single yeast cell with an ultra-sensitive radio frequency sensor, Lab on a Chip, 10, pp. 553-555, 2010.

[5] Y. Ning et al, 'Broadband electrical detectino of individual biological cells,' T-MTT, 2014, 62, 1905-1911.

[6] J. Leroy et al., "High frequency microfluidic biosensors for intracellular dielectric spectroscopy, IMS 2015.

[7] W. Chen, D. Dubuc, K. Grenier, "Microwave dielectric spectroscopy of a single biological cell with improved sensitivity up to $40 \mathrm{GHz}$,' IEEE IMS (2015). 